\title{
Chanson, circulation et mémoire: l'exemple de Sou eu mesmo, o trocado - Non, je ne regrette rien de Maria Bethânia
}

Canção, circulação e memória: o exemplo de Sou eu mesmo, o trocado - Non, je ne regrette rien de Maria Bethânia

Song, circulation and memory: the example of Maria Bethânia's Sou eu mesmo, o trocado - Non, je ne regrette rien

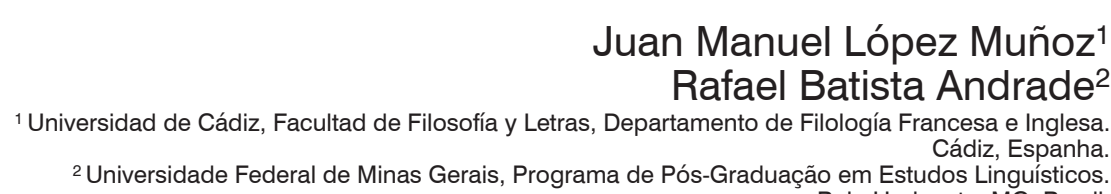

Manuel Lôpez Muñoz

Cádiz, Espanha. Belo Horizonte, MG, Brasil.

Professeur à la la Faculté des Lettres de l'Universite de Cadix, oú il est membre du groupe de re cherche HUM160 "Estudios de Filologia francesa". II est membre fondateur du groupe de recherche international en Analyse du Discours "Ci-DI" toires de "Pragmatique historique du francais" de Université Complutense de Madrid (Espagne) "Langage et Traitement Automatique" de l'Universite de Sfax (Tunisie). Ses publications portent notamment sur les notions de "circulation des discours", "postures énoncialves" el "dentie" dans un corpt. de textes variess, médievauux et contemporains. E-mail: imanuel.lopez@uca.es

Doctorant en Linguistique à l'Université Fédérale de Minas Gerais avec stage doctoral à l'Université de Cadix (boursier Fundación Carolina) et à l'Université Paris-Sorbonne - Paris IV (boursier CAPES PDSE: 88881.132934/2016-01). Professeur a l'Institut Federal de Minas Gerais campus Congonhas. de discurso nas cancōes-poemas de Maria Bethân de discurso nas cançóes-poemas de Maria E-mail: rafael.andrade@ifmg edu b.
RESUME: Dans cette étude, nous présentons une analyse de la chanson dialogique Sou eu mesmo, o trocado - Non, je ne regrette rien de Maria Bethânia (2016) à partir de la théorie de la circulation des discours développée par le groupe Ci-Dit (notamment LOPEZ-MUÑOZ; MARNETTE; ROSIER, 2009) et de l'apport maingueneaunien en analyse du discours. Notre étude révèle l'efficacité de ces théories pour la (re)catégorisation discursive de textes génériquement hybrides.

Mots clés: Mémoire; Discours; Paratopie; Scénographie; Chanson; Poésie.

RESUMO: Neste trabalho, apresentamos uma análise da canção dialógica de Maria Bethânia Sou eu mesmo, o trocado - Non, je ne regrette rien (2016) a partir da teoria da circulação discursiva desenvolvida pelo grupo Ci-Dit (em particular LÓPEZ-MUÑOZ; MARNETTE; ROSIER, 2009) e da contribuição de Maingueneau à análise do discurso. Nosso estudo revela a eficácia dessas teorias para a (re)categorização discursiva de textos genericamente híbridos.

Palavras-chave: Memória; Discurso; Paratopia; Cenografia; Canção; Poesia.

ABSTRACT: In this paper, we present an analysis of Maria Bethânia's dialogic song Sou eu mesmo, o trocado - Non, je ne regrette rien (2016) based on the theory of speech circulation developed by the group Ci-Dit (especially LOPEZ-MUNOZ; MARNETTE; ROSIER, 2009) and the Maingueneaunean contribution to discourse analysis. Our study reveals the effectiveness of these theories for a discursive (re)categorization of generically hybrid texts. Keywords: Memory; Discourse; Paratopy; Scenography; Song; Poetry. 


\section{Introduction}

n 2016, est apparu au Brésil le double album Abraçar e agradecer de Maria Bethânia ${ }^{1}$, issu de deux concerts célébrés les 7 et 8 août 2016 à l'aréna HSBC de São Paulo en commémoration des 50 ans de carrière et du $70^{\mathrm{e}}$ anniversaire de cette artiste. L'album contient des chansons inoubliables de l'inventaire de Bethânia ainsi que des chansons inédites composées spécialement pour elle, à l'occasion de cette célébration, par Nelson Motta, Paulo Cesar Pinheiro et Dori Caymmi entre autres. Certaines chansons y sont couplées à des poèmes de Fernando Pessoa, Clarice Lispector, Wally Salomão et d'elle-même. Le DVD 1 contient le concert et le DVD 2 propose des bonus. Notre étude est centrée sur le premier volume, en particulier sur la piste 37, intitulée "Non, je ne regrette rien", qui nous a particulièrement intrigués à cause de son caractère hybride ${ }^{2}$ : en effet, la chanson y est additionnée d'un poème de Alvaro de Campos (hétéronyme de Fernando Pessoa), au début, et d'un monologue de l'interprète, à la fin, selon la structure suivante:

1을 Dramatisation du poème "Sou eu mesmo, o trocado" (extrait de Sim, sou eu, eu mesmo, tal qual resultei de tudo) d'Alvaro de Campos, écrit en 1931 (publié en 1944).

\footnotetext{
${ }^{1}$ Née en 1946 au Brésil, Maria Bethânia a commencé sa carrière de chanteuse de musique populaire brésilienne (MPB) en 1963 dans la pièce de théâtre "Boca de Ouro" de Nelson Rodrigues. Son premier album est sorti en 1967. Le concert historique au "Canecão" en 2001 pour commémorer ses trentecinq ans de carrière représente incontestablement une étape dans la consolidation du statut de Maria Bethânia dans le cadre de la MPB. Elle y a réuni les principaux artistes de la MPB comme invités illustres: Adriana Calcanhoto, Ana Carolina, Arnaldo Antunes, Branco Melo, Caetano Veloso, Carlos Lyra, Chico Buarque, Chico César, Renato Teixeira, etc. Son nouvel album, Abraçar et agradecer est son 52 ème album en 50 ans de carrière.

2 Nous l'appellerons par la suite sous le titre de "sou eu mesmo - Non, je ne regrette rien" pour éviter l'ambiguité référentielle. Cette chanson est accessible en ligne en bonne qualité sur la page Facebook de culturando-se, sur le lien: https://www.facebook.com/cultuados/videos/1128517687196912. Il est à noter qu'elle fait également partie de la bande sonore du deuxième volumen de l'abum, contenant une vidéo de l'exposition d'art plastique "Maria de todos nós". Il s'agit d'une exposition qui a réuni des ouvres inédites de cent soixante artistes plastiques, musiciens, écrivains, photographes, amis et fans autour de l'imaginaire de Maria Bethânia.
}

$2^{\text {}}$ Chanson "Non, je ne regrette rien" (enregistrées pour la première fois par Édith Piaf le 10 novembre 1960, avec paroles de Michel Vaucaire et la musique de Charles Dumont ${ }^{3}$ ).

3o Monologue de Maria Bethânia.

L'intérêt de cette étude dans le cadre du présent numéro de Letrônica est double, étant donné qu'elle vise un cas remarquable de la fonction mémorielle des discours liée aux problématiques de l'interdiscours (fondé sur la dynamique entre genres de discours) et de l'interculturel (fondé sur l'altérité des mémoires culturelles ${ }^{4}$ ). De plus, la chanson analysée Sou eu mesmo, o trocado/Non, je ne regrette rien place la démarche de notre recherche à la croisée de plusieurs propositions théoriques ayant trait à la thématique de ce volume, notamment celles concernant l'étude des pratiques langagières relevant du dialogisme et de la circulation des discours.

L'hypothèse ici défendue est que la pratique, courante chez Maria Bethânia, de la récitation de poèmes dans le contexte de ses performances de musique populaire brésilienne (MPB) ne doit pas être considérée comme une forme particulière de citation mais comme une stratégie qui réussit à engendrer effectivement un nouveau genre de discours. Ce nouveau genre

${ }^{3}$ Charles Dumont a composé quarante titres en trois ans pour Edit Piaf. Et ce n'est pas sans doute par hasard qu'il a intitulé son livre autobiographique Non, je ne regrette toujours rien (DUMONT, 2012). En ce qui touche à nos objectifs dans cette étude, il est intéressant de noter cette citation extraite de l'autobiographie de ce compositeur, où il révèle les clés de son succès musical: "Il a suffi de cet éclair qui s'appelle Non, je ne regrette rien [de Michel Vaucaire] pour vivre une nouvelle vie. Le miracle de cette chanson est d'avoir su harmoniser une musique pleine de colère à des mots d'acceptation et d'espoir. Édith Piaf attendait-elle depuis longtemps cette nouvelle force ? En tout cas, avec cet instinct prémonitoire qui la caractérise, elle a immédiatement deviné ce qu'il en serait pour elle et pour moi (DUMONT, 2012, p. 121).

4 Nous empruntons ce terme à l'anthropologue culturel Jan Assmann, qui le défini comme "Une mémoire longue qui conserve des lignes et qui garantit leur actualisation à l'aide des moyens les plus divers, du rituel au symbole, des images/ et qui garantit leur actualisation à "aide des moyens les plus divers, du rituel au symbole, des images/

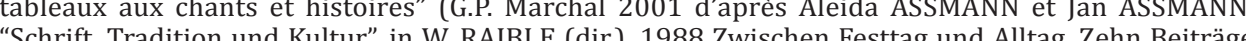
zum Thema, "Mündlichkeit und Schriftlichkeit", Tübingen). 
de chanson dialogique est caractérisé, selon nous, par une sublimation de l'énonciateur lequel, dissout et réduit en ses principes au moyen d'une paratopie identitaire, spatiale et temporelle résultant du gommage des repères énonciatives et du brouillage des frontières des genres, peut agir, déclamer ou chanter, libéré des contraintes, et changer de langue et même de sexe sans nuire à la cohérence de son point de vue. Le processus de sublimation de l'énonciateur, pour qu'il soit réussi, implique l'investissement affectif, mémoriel et cognitif des destinataires.

\section{La circulation des discours: territoires, médiateurs et mémoire(s)}

Le premier point à considérer par rapport aux conditions de production et de mise en scène de la chanson dialogique Sou eu mesmo, o trocado/Non, je ne regrette rien est celui de la circulation des discours.

Nous considérons la circulation des discours (LOPEZ-MUÑOZ, 2008, 2014; LOPEZ-MUÑOZ; MARNETTE; ROSIER, 2004, 2006, 2009) comme un ensemble de mécanismes d'appropriation et de re-énonciations de discours par des médiateurs ou "agents de circulation". Ces médiateurs de discours agissent à titre institutionnel et/ou personnel, que ce soit régulièrement, suivant un plan programmé de circulation (agents médiatiques, académiques, etc.) ou, au contraire, occasionnellement et de manière spontanée, en passant par toute une panoplie de situations mixtes entre ces deux pôles.

De façon générale, la circulation du discours, qu'elle soit programmée ou spontanée, contrainte ou volontaire, peut résulter du déplacement du locuteur (les discours des exilés, par exemple) ou bien, le plus souvent, du déplacement du discours lui-même, qui devient objet "circulant", relayé par d'autres locuteurs. En ce sens, le discours rapporté constitue une forme primordiale, linguistiquement marquée, de circulation, mais pas la seule.
La notion de circulation de discours présuppose l'existence de territoires de circulation (LOPEZ-MUÑOZ, 2010): ces territoires ne doivent pas être confondus ni avec les lieux d'énonciation ni avec l'espace discursif de chaque genre, ni enfin avec le territoire national-géographique de chaque langue, qui sont tous des espaces fixes, aux limites stables. Le territoire des "circulants" est caractérisé par l'impossibilité de l'ancrage et par la mouvance permanente des frontières, c'est un non-lieu et en ce sens il est comparable à la notion de paratopie développée par Dominique Maingueneau, sur laquelle nous reviendrons plus bas. Moins un discours est énonciativement embrayé, plus il est apte à devenir "circulant" et donc à traverser des territoires: c'est surtout le cas des proverbes et de beaucoup d'aphorismes, mais aussi de beaucoup de poèmes et des chansons, comme dans la présente étude.

Il est enfin important de distinguer la circulation des discours d'autres phénomènes plus ou moins mécaniques de propagation de mots ou d'idées: nous tenterons de montrer ici, à la suite de López-Muñoz (2014) que la circulation de discours est quelque chose de vivant et d'agissant, à la fois transitif (un discours est transféré à un autre) et génératif (le discours relayé génère un autre discours), mobilisant les savoirs, les souvenirs, les comportements et les émotions des participants à l'acte de mise en circulation.

Cela dit, et pour revenir à notre corpus d'étude, nous entendons la reénonciation du poème portugais (Sou eu mesmo o trocado) et de la chanson française (Non, je ne regrette rien) par Maria Bethânia dans le cadre d'un concert de musique populaire brésilienne comme un cas exemplaire relevant de la circulation des discours. Celle-ci a opéré (comme d'habitude elle opère d'ailleurs) à plusieurs niveaux. Ces deux textes ont dû, en effet, traverser des frontières culturelles, géographiques et temporelles en plus d'outrepasser des limites entre genres de discours avant de se matérialiser au récital de 
Maria Bethânia au Brésil. Il va sans dire que cette chanteuse n’a pas été la première ni la seule médiatrice de ces deux textes. Bien évidemment, ils circulaient déjà depuis longtemps et sur de larges territoires. Ces deux "circulants", partant de cadres institutionnels différents, ont utilisé plusieurs médiateurs aux différents rôles tout au long de leur circulation, ce qui n'est pas sans conséquence, comme on le sait, sur leurs respectives conditions de diffusion et de réception (LOPEZ-MUÑOZ, 2010) à chaque (re)énonciation.

Si toute re-énonciation est, chaque fois, un acte singulier renouvelant l'énoncé relayé au point de le faire redevenir token (même au cas où il s'agirait d'une répétition à l'identique), la singularité à cette occasion tient à la manière comment Maria Bethânia réussit à donner à son acte de mise en circulation de discours une triple fonction à la fois transitive, rénovatrice et générative, à travers diverses stratégies, que nous présentons ensuite.

La fonction transitive dérive de la reprise de "circulants" et de l'inscription de ceux-ci dans une nouvelle situation d'énonciation. Ces "circulants" sont, dans le cas de la chanson dialogique de Maria Bethânia, le poème de Pessoa et la chanson de Piaf. La chanteuse les a transférés à la situation d'énonciation de son récital de la manière suivante: Le concert a une durée de 1:52:10 (une heure, 52 minutes et 10 secondes). À 01:33:18, après les applaudissements consécutifs à la chanson Eu te desejo amor (item 36), Maria Bethânia commence à déclamer en portugais le poème Sou eu mesmo, ou trocado de Pessoa, sans accompagnement instrumental. À 1:33:52 commence à sonner le thème musical de la chanson d'Edit Piaf, alors que la chanteuse est en train de prononcer les derniers vers du poème. À peine terminé le poème, Maria Bethânia se met aussitôt à chanter en français Non, je ne regrette rien. Après cette chanson, sans transition et sans que le thème musical de Piaf cesse de sonner, elle revient au portugais avec le monologue Não me arrependo de nada, une sorte de journal intime dramatisé, construit sur la synthèse des idées principales des deux "circulants" qu'elle prend en charge à titre personnel (nous y reviendrons). La chanson se clôt à 1:37:15 avec des applaudissements et les remerciements de l'interprète.

Quant à la fonction rénovatrice (LOPEZ-MUÑOZ et al., 2009), elle concerne la manière comment le sens et la forme des "circulants" ont été traités lors de leur re-énonciation. Ainsi, la circulation est "conservatrice" si le circulant est transféré sans modification de sens ni de forme. Au contraire, la circulation est (ré)novatrice lorsqu'elle transforme le circulant au point que celui-ci devient source de co-construction de discours et d'hybridation des points de vue. Nous soutenons que l'interprète de la MPB met en place une circulation rénovatrice de sens et forme du poème de Pessoa et de la chanson de Piaf. Du point de vue formel, cette circulation est textuellement marquée par un positionnement artistique propre qui se manifeste dans des opérations de coupe (omissions de certaines parties du poème de Pessoa) et de collage associées au processus de mise en scène et de dramatisation. Nous présenterons cela de manière détaillée plus bas.

La fonction générative de la circulation concerne enfin la participation de celle-ci à la configuration de nouveaux genres des discours via la transgression d'espaces discursifs frontaliers. Dans le cas spécifique qui nous occupe, le processus de re-énonciation se double d'une interversion $(o$ trocado) des propriétés génériques des deux circulants: d'un côté, le poème écrit est oralisé, dramatisé, accompagné de musique dans un concert; de l'autre côté, la chanson de Piaf dérive en journal intime, prose dramatisée. Ainsi, la chanson dialogique résultante, fabriquée dans un cadre hautement légitimant, apparait comme le produit d'un nouveau genre de discours hybride faisant appel à la mémoire interdiscursive.

En effet, une caractéristique remarquable du poème dont il est question dans le cadre de notre étude est que, appartenant à la production littéraire d'un poète qui fait autorité dans la société portugaise - et dont la transcendance est mondialement reconnue -, il joue un rôle de "discours 
constituant" (MAINGUENEAU, 2006) à l'égard de la chanson dialogique qu'il introduit. Il légitime et donne sens à la chanson de Maria Bethânia, avec un fonctionnement assez singulier. Car il s'avère que le texte ainsi garanti contient aussi un autre discours constituant, relevant cette fois de l'espace institutionnel de la chanson française: les paroles de Non, je ne regrette rien. Deux temps historiques, deux langues, deux institutions, orchestrés au présent de l'énonciation du concert de 2016 à HSBC Arena de São Paulo pour la commémoration du $50^{\mathrm{e}}$ anniversaire de la carrière musicale la chanteuse brésilienne Maria Bethânia. La chanson se pose ainsi en lieu de rencontre de trois mémoires (culturelles, discursives), à partir d'un embrayage paratopique basé sur le paradoxe de l'impossible appartenance des discours (littéraires et autres) aux circonstances (personnes, temps, lieux, langues) qui les font émerger.

\section{Paratopie}

Maingueneau (2014) a souligné quatre axes majeurs de la paratopie (qu'il appliquait au champ littéraire mais qui, selon nous, peut concerner plusieurs autres champs de la création artistique et notamment la chanson): identitaire, spatial, temporel et linguistique. Dans tous les cas la paratopie caractérise ce discours qui, tout en état un produit singulier, événementiel, est marqué par le collectif vis-à-vis duquel le sujet (l'auteur dans le cas de la littérature, Bethânia dans notre cas) se positionne: c'est un processus impliquant la construction d'une singularité discursive qui prend paradoxalement appui sur des discours "déjà-là" ("circulants") et sur des pratiques discursives institutionnalisées, bref "sur des schémas partagés par la collectivité dont on attend la reconnaissance" (MAINGUENEAU, 2004, p. 14). La paratopie signale ainsi la négociation entre le moi et non-moi (les autres comme moi mais aussi les autres qui ne sont pas/ne parlent pas comme moi ou encore qui ne parlent pas la même langue que moi), le lieu et le non-lieu, le temps et le non-temps, et donc la possibilité de rester dans les marges, dans les périphéries, dans l'entre-deux, de manière parasitaire, en échappant à ces dialectiques qui ne sont qu'apparentes et qui permettent justement des pratiques de mélange et d'hybridation, comme le fait justement Bethânia.

Nous soutenons l'idée que, dans la chanson Sou eu mesmo - Non, je ne regrette rien, Maria Bethânia apparaît comme un sujet sublimé, paratopique, comme quelqu'un qui n'a pas "lieu d'être" (ou plutôt qui ne veut pas avoir lieu d'être) et qui construit son chant - et donc son point de vue marginal, parasitaire - à travers justement la volonté de ne pas s'assigner une identité unique, une seule langue, une voix monophonique et une place stable dans l'espace-temps institutionnel de la chanson populaire brésilienne.

Maria Bethânia, le sujet pluriel qu'elle est, chanteuse, actrice, metteur en scène, (se) déclame et (se) chante à travers un "je" qui est à la fois Alvaro de Campos, Fernando Pessoa, Michel Vaucaire, Charles Dumont et Édith Piaf, sans jamais cesser pour autant d'être elle-même, celle qu'elle est au moment de la performance et aussi celle qu'elle a été auparavant, durant sa longue carrière. Le brouillage des voix obtenu par l'omission des repères énonciatifs (elle prend soin de ne pas expliciter les sources des "circulants" dont elle s'approprie, amplement connues par ailleurs) constituent un des éléments principaux d'une stratégie discursive consciente destinée à se construire l'image d'une chanteuse qui se réinvente en explorant les limites des identités, des espaces et des genres de discours qu'elle fonde et qui fondent ses chansons.

Concernant l'axe spatial, Maria Bethânia réussit à mettre en question la propre place des deux arts dans la société: ceux de la chanson populaire et de la littérature légitimée. En participant à la circulation des poèmes de 
Pessoa avec la scénographie ${ }^{5}$ propre à la chanson populaire brésilienne, elle abolit les contraintes qui empêchent normalement la manifestation des deux arts ensemble en même temps et au même endroit. Nous pouvons dire selon les mots de Maingueneau (2016, p. 27) que Maria Bethânia « va de place en place sans jamais se fixer". De plus, en choisissant un poème de la littérature en langue portugaise (plus spécifiquement du Portugal) et une chanson française, elle propose son texte comme étant le lieu de rencontre, au Brésil, de deux langues autres, européennes (et donc de deux mémoires culturelles autres) preuve que son art se joue aussi des frontières identitaires, nationales et linguistiques.

Par rapport à la paratopie temporelle, Maria Bethânia construit sa chanson sur un embrayage temporel axé sur le présent de l'énonciation, qui s'avère être le non-temps caractéristique des actes commémoratifs. Ce temps présent, grâce à l'appareil formel de l'effacement énonciatif mis en œuvre (RABATEL, 2003; VION, 2001), fonctionne comme une faille à travers laquelle on peut accéder, sans rupture temporelle, au temps présent du poème de Pessoa, Sou eu mesmo (“je suis moi-même”), daté de 1931, en continuation avec le présent de la chanson française, je ne regrette rien, daté de 1961 et enfin avec celui de não me arrependo de nada qui parachève la chanson de Bethânia le jour du concert, daté de 2016. Finalement, étant donné que la chanson s'inscrit dans un acte organisé pour le $50^{\mathrm{e}}$ anniversaire de la carrière musicale de Maria Bethânia, le présent de son énonciation s'enrichit de passé, grâce à la dynamisation de la mémoire (ou plutôt des mémoires: affective, encyclopédique, culturelle, discursive...), individuelle et collective.

\footnotetext{
${ }^{5}$ Dans l'analyse du discours maingueneaunien (MAINGUENEAU, 2004, p. 190-202), la scène d'énonciation sous-tend une triple division: la scène englobante, la scène générique et la scénographie. La première correspond au type du discours (littéraire, philosophique, etc.). La deuxième désigne les conditions d'énonciation de certains genres de discours appartenant à un même type de discours (épopée, roman, conte, etc.). La dernière rend compte de la singularité énonciative; elle dépend des choix du locuteur, qui peut s'écarter ou non des modèles d'énonciation établis par les scènes englobantes et génériques.
}

Ces trois types de traits paratopiques se combinent, au plan discursif, avec un processus qui débute par la dramatisation du poème de Pessoa, et que l'on peut décrire de la manière suivante.

\section{Au plan discursif}

Tout d'abord, il convient de préciser, pour commencer, que la chanson objet de notre étude n'est pas le premier cas où Maria Bethânia joue un texte littéraire dans le but d'introduire ou de complémenter ses chansons, mais que cela fait partie d'une pratique récurrente chez cette interprète (ANDRADE, 2011, 2015, 2016). D'autre part, nous n'approfondirons pas ici sur la notion de "dramatisation", de peur de susciter plusieurs questions qui nous éloigneraient des objectifs qu’on s'est fixés: différences entre dramatisation, jeu, monologue, déclamation... Nous comprendrons simplement le concept de dramatisation, au sens large, comme la technique permettant de réciter une œuvre devant un public de manière à la rendre plus vivante, plus expressive. Cela nous permettra de faire ressortir quelques traces particulières des stratégies transgressives (transdiscursives) mises en place par Maria Bethânia.

La chanteuse brésilienne se présente comme une médiatrice qui joue sur le brouillage des frontières discursives, laissant entièrement au public la responsabilité de la reconnaissance des différentes scènes qui sous-tendent la chanson. Elle met ainsi en place une stratégie de circulation de discours en quelque sorte comparable à d'autres pratiques discursives dans le domaine d'arts comme la parodie et le pastiche, mais dont les effets recherchés sont certainement différents.

Ainsi, aux destinataires modèles de la chanson dialogique de Maria Bethânia sont imposées certaines contraintes herméneutiques. Tout d'abord, ils sont censés pouvoir distinguer lors de la performance le poème 
emprunté à Pessoa du texte de la chanson de Piaf, et celui-ci de l'élocution finale, et cela malgré l'absence de marques de transition et surtout malgré la musique qui connecte les trois parties. Par rapport à la chanson de Piaf, le destinataire idéal devrait pouvoir également en reconnaître le compositeur et le distinguer de sa célèbre interprète. Ainsi, dans le cas de Non, je ne regrette rien, le public devrait-il pouvoir discriminer la voix d'Édit Piaf et le point de vue de Michel Vocaire dans le timbre et la manière de chanter singuliers de Maria Bethânia. Quant à la reconnaissance de l'auteur du poème Sou eu mesmo, le public idéal devrait compter sur un savoir encyclopédique (MAINGUENEAU, 1984) lui permettant de distinguer Fernando Pessoa sous l'hétéronyme d'Alvaro de Campos. Les fans de Maria Bethânia savent sans doute que Fernando Pessoa est un des poètes préférés de la chanteuse brésilienne. Ils peuvent en effet se souvenir d'un CD dont tous les textes étaient de Fernando Pessoa et de ses hétéronymes (BETHANIA, 1997).

La reconnaissance de Fernando Pessoa comme auteur de Sou eu mesmo, o trocado se double d'un deuxième défi, car la question se pose si le poème a été intégralement reproduit ou bien manipulé.

En effet, la confrontation avec le texte original du poète fait apparaître des coupes ici et là et des arrangements qui méritent d'être examinées. Nous présentons dans le tableau qui suit les deux textes, à gauche l'original (où nous soulignons les vers ou morceaux de vers que Maria Bethânia a retenus pour son récital, le reste constituant la partie non dite) et, dans la colonne à droite, le texte du poème dramatisé par la chanteuse. En note, page suivante, le lecteur pourra trouver la traduction française du poème complet.

\section{TEXTE ORIGINAL DE FP \\ Sim, sou eu, eu mesmo, tal qual resultei de tudo, Espécie de acessório ou sobressalente próprio, Arredores irregulares da minha emoção sincera, Sou eu aqui em mim, sou eu.}

Quanto fui, quanto não fui, tudo isso sou.

Tuanto quis quanto não quis, tudo isso me forma.

Quanto amei ou deixei de amar é a mesma saudade em mim.

E, ao mesmo tempo, a impressão, um pouco inconseqüente, Como de um sonho formado sobre realidades mistas,

De me ter deixado, a mim, num banco de carro elétrico,

Para ser encontrado pelo acaso de quem se lhe ir sentar em cima.

E, ao mesmo tempo, a impressão, um pouco longínqua,

Como de um sonho que se quer lembrar na penumbra a que se acorda,

De haver melhor em mim do que eu.

Sim, ao mesmo tempo, a impressão, um pouco dolorosa,

Como de um acordar sem sonhos para um dia de muitos credores,

De haver falhado tudo como tropeçar no capacho,

De haver embrulhado tudo como a mala sem as escovas,

De haver substituído qualquer coisa a mim algures na vida.

Baste! É a impressão um tanto ou quanto metafísica,

Como o sol pela última vez sobre a janela da casa a abandonar

De que mais vale ser criança que querer compreender o mundo -

A impressão de pão com manteiga e brinquedos

De um grande sossego sem Jardins de Prosérpina,

De uma boa-vontade para com a vida encostada de testa à janela,

Num ver chover com som lá fora

E não as lágrimas mortas de custar a engolir.

Baste, sim baste! $\underline{\text { Sou eu mesmo, o trocado }}$

0 emissário sem carta nem credenciais.

0 palhaço sem riso, o bobo com o grande fato de outro

Como chocalhos pequenos de uma servidão em cima.

Sou eu mesmo, a charada sincopada

Que ninguém da roda decifra nos serões de província.

\section{TEXTE REPRODUIT PAR MB}

Sou eu mesmo,

Sou eu mesmo, o trocado,

Sou eu mesmo, o trocado,

0 emissário sem cartas nem credenciais,

0 palhaço sem riso, o bobo com o grande fato de outro,

Sou eu mesmo, a charada sincopada

Que ninguém da roda decifra nos serões da província.

Quanto fui, quanto não fui, tudo isso sou.

Quanto quis, quanto não quis, tudo isso me forma.

Quanto amei ou deixei de amar é a mesma saudade em mim.

Sou eu mesmo, a charada sincopada

Que ninguém da roda decifra nos serões de província 
Compte tenu des nombreuses omissions et des arrangements, on peut se poser la question de l'intention de Maria Bethânia. Peut-on y voir une manœuvre de désacralisation de la poésie légitimée ou bien (ou surtout) une invitation au jeu de la récupération mémorielle?

NOTE: La version française du poème - traduite du portugais par Patrick Quillier avec la participation de Maria Antónia Câmara Manuel, est la suivante:

Oui, je suis moi, moi-même, tel et quel j'ai résulté de tout,/ Espèce d'accessoire ou de rechange spécifique,/ Banlieueur irrégulière de mon émotion sincère, / Je suis moi ici en moi, je suis moi. / / /Tout ce que j'ai été, tout ce que je n'ai pas été, je suis tout ça./ /Tout ce que j'ai voulu, tout ce que je n'ai pas voulu, tout ça me forme. / Tout ce que j'ai aimé ou cessé d'aimer, c'est la même saudade en moi. / / /Et, en même temps, l'impression, un peu inconséquent, / Comme d'un rêve se formant sur des réalités mêlées, / De m'être laissé moi-même sur une banquette de tram, / Afin d'être trouvé par le hasard de qui viendrait s'asseoir dessus. / / / Et, en même temps, l'impression, un peu lointaine, / Comme d'un rêve dont on veut se souvenir dans la pénombre où l'on s'est réveillé, / Qu'il y a en moi meilleur que moi-même. / / /Et, en même temps, l'impression, un peu douloureuse, / Comme d'un réveil sans rêves dans un jour riche en créanciers. / D'avoir tout raté comme on trébuche sur le paillasson, / D'avoir tout empaqueté comme la valise sans les brosses, / D'avoir mis quelque chose à ma place quelque part dans la vie. / / /Suffit ! C'est l'impression un tantinet métaphysique, / Comme le soleil pour la dernière fois sur la fenêtre de la maison qu'on va abandonner, / Qu'il vaut mieux être enfant que de vouloir comprendre le monde - / Limpression de pain beurré et de jouets/ D'une grande paix sans Jardins de Proserpine, / D'une bonne volonté envers la vie la tête appuyée sur la fenêtre, / À voir pleuvoir dans un bruissement dehors/ Et non les larmes adultes de ce qui reste en travers de la gorge. / / /Suffit, c'est ça, suffit ! Je suis le même, l'interverti, / L'émissaire sans missive ni lettres de créance, / Le clown sans rire, le bouffon dans le trop grand costume d'autrui, / Et les clochettes lui tintent sur la tête/ Comme petites sonnailles d'une servitude pesant sur lui. / / /Je suis le même, la charade syncopée/ Que personne dans le petit cénacle ne déchiffre lors des soirées de province. / / /Je suis le même, quel réconfort! // (PESSOA, 2001, p. 256-257).
Le premier aspect à considérer est celui de titre de la chanson, sachant que le poème de Pessoa n'en avait pas originairement. Chez les éditeurs de poésie, une certaine tendance s'est imposée de nommer les poèmes à partir du premier vers. Nous avons trouvé cette pratique dans les éditions consultées, celle de 2008 chez Companhia Editora Nacional à São Paulo, pour l'édition brésilienne et celle de Christian Bourgeois, à Paris 2001, pour la traduction française ${ }^{6}$. Dans le premier cas, les éditeurs ont utilisé le titre Sim, sou eu, eu mesmo, tal qual resultei de tudo, c'est-à-dire que le titre est composé du premier vers complet du poème. Dans le second cas, on constate le titre suivant: Oui, je suis moi, moi-même; c'est-à-dire que le titre y est une forme abrégée du premier vers du poème.

Maria Bethânia transgresse cette tradition en faisant commencer sa chanson par le second hémistiche du vers 28: Sou eu mesmo, o trocado. De plus, notons la différence fondamentale que, dans le livret du CD, c'est le titre de la chanson Non, je ne regrette rien qui prend les devants.

Précédemment, nous avons indiqué qu'au moyen de la dramatisation, le poème de Pessoa subit un remplacement de sa forme de texte littéraire (modalité écrite) pour une autre à modalité orale. Dans le cas spécifique de Sou eu mesmo, o trocado la transformation formelle concerne également son étendue et sa structure. Sans cesser d'être reconnaissable comme étant le poème de Pessoa, il acquiert dans sa re-énonciation par Bethânia une structure différente de l'original; il y (ré)apparaît beaucoup plus court et avec un nouveau début, avec un nouveau milieu et surtout avec une nouvelle fin du fait de l'omission par Bethânia du dernier vers de Pessoa et de l'empiètement du poème sur le "terrain" de la chanson d'Édit Piaf. Or sur cet aspect structurel, Bakhtine lui-même (1984) a justement remarqué l'importance de délimitation entre le début et la fin dans la construction

${ }^{6}$ Le CD/DVD de Maria Bethânia ne donne pas la référence à l'édition utilisée. 
compositionnelle du texte comme critère pour la définition des genres du discours. La nouvelle forme sous laquelle le poème de Pessoa est reénoncé peut ainsi être analysée, selon nous, comme un des traits discursifs de ce nouveau genre de chansons pratiqué par Bethânia et que nous avons convenu d'appeler chanson dialogique ${ }^{7}$.

Après avoir constaté ces changements au niveau de la structure du poème, d'autres questions s'imposent concernant toujours les modifications réalisées par la chanteuse à partir de l'original de Pessoa. En effet, on peut se demander pourquoi a-t-elle choisi ces parties soulignées à la place d'autres extraits potentiels de ce poème. Deux observations principalement nous permettront de mieux comprendre la circulation rénovatrice de sens et forme mise en place par la Maria Bethânia.

D'une part, la chanteuse brésilienne a sans doute récité ces parties du poème qu'elle a considérées comme étant à reprendre, c'est-à-dire particulièrement détachables ${ }^{8}$ (au sens de MAINGUENEAU, 2012), celles qui se prêtent mieux à l'extraction pour l'usage dans le nouveau contexte de sa chanson à elle, que ce soit grâce à la position saillante des vers choisis dans le texte source (sou eu mesmo...), soit à la structure prégnante de leur signifiés ou signifiants (quanto fui... quanto quis, quanto amei...), ou encore par leur autonomie référentielle (a charada sincopada que ninguém da roda decifra...). Ce sont, après tout, justement ces éléments détachables qui permettront au public connivent de récupérer, par le recours à leurs souvenirs, la totalité du poème, ou du moins à y reconnaître les manipulations opérées. Dans cette

7 Dans des travaux antérieurs (notamment ANDRADE, 2016) nous avions proposé le terme de "chanson polyphonique"; nous lui préférons désormais celui de "chanson dialogique" pour éviter toute possible identification non souhaitable avec les chansons polyphoniques du Moyen Âge (Ars nova Guillaume

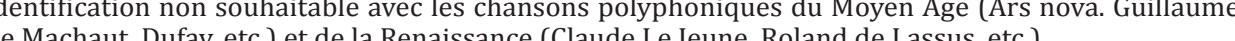
de Machaut, Dufay, etc.) et de la Renaissance (Claude Le Jeune, Roland de Lassus, etc.)

Selon Maingueneau, la " détachabilité » fait écho, au plan énonciatif, à la " surassertion », entendue comme opération énonciative par laquelle le locuteur montre son point de vue dominant en donnant propre texte. Nous y reviendrons pour apporter quelques nuances au cas de la chanson étudiée. perspective, l'inscription du poème de Pessoa dans la chanson de Maria Bethânia peut être assimilé à l'interdiscours, en ce sens qu'il ne s'agit pas uniquement d'énoncés matériellement repérables mais aussi, et surtout, d'un discours autre, poétique, portugais, qui "sonne et trébuche" (PAVEAU, 2010, p. 8) dans la chanson de la Brésilienne.

D'autre part, les suppressions suivent la propre logique de la chanson dialogique telle que nous l'avons définie ailleurs ${ }^{9}$, conditionnée par les contraintes des nouvelles conditions d'énonciation dans lesquelles le texte repris s'inscrit. Ainsi, les thèmes incompatibles avec la chanson de Maria Bethânia ont-ils été supprimés au profit de ceux qui, au contraire, nourrissent et enrichissent l'idée de l'acceptation inconditionnelle de soi qui est à centre de Non, je ne regrette rien. Le texte de Pessoa prend donc, au-delà d'une nouvelle forme comme nous avons vu, de nouveaux sens qui s'originent à partir des manœuvres de coupes et de raccommodage réalisées par Maria Bethânia. On peut y constater, à la suite de Maingueneau (2014, p. 126) comment, à travers “les parcours qu'[elle] y trace et ceux qu'[elle] exclut, [la chanteuse brésilienne] indique quel est pour [elle] l'exercice légitime" de son art.

On sait que le thème majeur du poème de Pessoa est la pensée et l'acceptation, (que remédio!) de sa propre existence ${ }^{10}$, en bonne harmonie avec celui de la chanson de Piaf. Or ce poème aborde d'autres thèmes plus ou moins secondaires qui, tout en étant cohérents avec le projet pessoien, se sont avérés incompatibles avec la chanson de Bethânia. Parmi ces thèmes supprimés, le principal est celui de la nostalgie du regard enfantin, innocent, sur le sens de la vie, que l'auteur développe dans les vers 20 à 27:

9 Voir n. b. p. no 12 ici même.

${ }^{10}$ De surcroit, ce sujet est l'axe central dans l'œuvre de Pessoa-Álvaro de Campos: "Sa poésie est le livre de bord de l'interminable voyage qu'il réalise dans l'intérieur de lui-même, comme si toute elle, vers à vers, ait compromis à répondre à une seule question essentielle, quasi toujours implicite: Qui suis-je?" (MOISES, 1998, p. 29) 
Baste! É a impressão um tanto ou quanto metafísica,

Como o sol pela última vez sobre a janela da casa a abandonar,

De que mais vale ser criança que querer compreender o mundo -

A impressão de pão com manteiga e brinquedos

De um grande sossego sem Jardins de Prosérpina,

De uma boa-vontade para com a vida encostada de testa à janela,

Num ver chover com som lá fora

E não as lágrimas mortas de custar a engolir.

Pareillement, Maria Bethânia a évité (devrait-on dire balayé?), au moyen de ses coupes, le thème de l'impression" qui débute et fonde les strophes 3 à 6 de Pessoa, sans doute à cause des connotations de trouble, de confusion et de sensation superficielle liées à ce terme: a impressão, um pouco inconseqüente (v. 8), a impressão, um pouco longínqua (v. 12), a impressão, um pouco dolorosa (v. 15), a impressão um tanto ou quanto metafísica (v. 20) ...

$\mathrm{Au}$ plan énonciatif, il résulte des suppressions qui précèdent un effet de point de vue surplombant (RABATEL, 2009), dont le caractère dominant peut être aisément reconnu par les destinataires: la chanteuse se présente comme quelqu'un qui s'autorise à s'approprier les vers de Pessoa, pour en fabriquer autre chose. Elle réussit, en effet, à dégager les grandes lignes du poème de Pessoa et, ce faisant, elle se construit à la fois une posture intellectuelle et une sorte de magistère artistique et moral. Comme le poète, Bethânia est "tout ce qu'elle a été et tout ce qu'elle n'a pas été". Aussi la chanson dialogique est-elle tout ce qu'elle dit et tout ce qu'elle omet.

\section{Au plan énonciatif}

Comme nous avons signalé plus haut, dès le début de la chanson dialogique jusqu'à la fin, la chanteuse a pris soin de brouiller les traces linguistiques permettant de distinguer les différents énonciateurs convoqués par elle: le poète, le compositeur, le parolier, la chanteuse française..., au moyen d'une habile gestion de l'appareil formel de l'effacement énonciatif
(RABATEL, 2003; VION, 2001). Selon Rabatel (2003), il existe trois possibles effets pragmatiques issus de l'effacement énonciatif: ce sont les effets de surénonciation, de coénonciation et de sousénonciation. Nous avons déjà mis en avant l'effet surénonciatif, surassertif, obtenu de la pratique du façonnage du point de vue de Campos-Pessoa à l'avantage de celui de l'interprète.

Nous voudrions maintenant y apporter une nuance que nous considérons importante, à partir d'un concept que nous avons introduit dans un travail antérieur (LOPEZ-MUÑOZ; ROMERAL, 2006), celui d'échoénonciation. Il désigne une posture intermédiaire entre la coénonciation et la surénonciation, impliquant une fusion totale de deux points de vue et la prise en charge des propos de l'autre sur la base d'une appropriation, à l'avantage propre, des paroles et de la position sociale d'une autorité reconnue. C'est une forme particulière de reprise échoïque qui partage avec l'écho la répétition plus ou moins littérale des signifiants, mais qui se distingue de celui-ci en ce sens qu'il n'opère jamais dans l'immédiateté de l'interaction, mais sur le mode de la reprise de "circulants" (de discours en circulation) faisant partie du patrimoine discursif d'une communauté. Bethânia s'approprie les vers (certains vers) de Pessoa, qui viennent corroborer sa chanson (et non pas l'inverse), au point même d'occuper l'identité du poète en s'emparant de son pronom "eu". C'est un "trocado" énonciatif, une sorte de travestissement énonciatif: la chanteuse s'autodésigne "eu mesmo", c'est-à-dire elle est le poète même. Notons qu'elle ne dit pas "eu mesma", au féminin. En re-énonçant ainsi les propos du poète, elle transgresse à la fois les limites des identités et la binarité de genre, ce qui lui permet, par la suite, de poursuivre sans gêne sur le "je" féminin de la chanson de Piaf et de conclure sur son "je" à elle-même. Bethânia s'érige donc en énonciateure qui prend en charge deux points de vue consécutifs constituant, en fin de compte, un même point de vue malgré les différences de genre, de langue, de discours et de mémoires (portugaise, française, brésilienne). 
Figure 1 - Le travestissement énonciatif dans la chanson dialogique

Énonciateur du poème

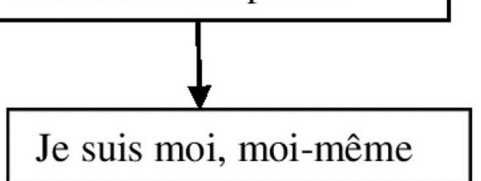

Je suis moi, moi-même

ÉNONCIATEUR DE LA CHANSON DIALOGIQUE

Dans les extraits suivants, nous avons signalé en caractères gras les marques de première personne pour faire ressortir comment ce jeu du travestissement énonciatif, aux effets de surassertion échoénonciative, vertèbre la chanson dialogique. Les soulignés indiquent les parties du texte accompagnées de la musique instrumentale de Non, je ne regrette rien:

\section{Sou eu mesmo}

Sou eu mesmo, o trocado,

Sou eu mesmo, o trocado,

0 emissário sem cartas nem credenciais,

O palhaço sem riso, o bobo com o grande fato de outro,

Sou eu mesmo, a charada sincopada

Que ninguém da roda decifra nos serões da província.

Quanto fui, quanto não fui, tudo isso sou.

Quanto quis, quanto não quis, tudo isso me forma.

Quanto amei ou deixei de amar é a mesma saudade em mim.

Sou eu mesmo, a charada sincopada

Que ninguém da roda decifra nos serões de província

Non, rien de rien, non, je ne regrette rien

Ni le bien qu'on m'a fait, ni le mal

Tout ça m'est bien égal

Non, rien de rien, non, je ne regrette rien

C'est payé, balayé, oublié, je me fous du passé
Avec mes souvenirs j'ai allumé le feu

Mes chagrins, mes plaisirs

Le n'ai plus besoin d'eux

Oublié les amours avec leurs trémolos Balayé pour toujours

Je repars à zéro

Non, rien de rien,

Non, je ne regrette rien

Ni le bien qu'on m'a fait, ni le mal

Tout ça m'est bien égal

Non, rien de rien,

Non, je ne regrette rien

Car ma vie, car mes joies

Aujourd'hui ça commence avec toi

Não me arrependo de nada

Nem do bem que me fizeram, nem do mal

Tudo isso, tanto faz.

Não me arrependo de nada

Está pago, varrido e esquecido

Não estou nem aí para o passado.

Com as minhas lembranças, eu acendi o fogo

Minhas tristezas e meus prazeres, eu não preciso mais deles. Varridos os meus amores, com seus tremores

Varridos para sempre

Recomeço do zero.

Não me arrependo de nada

Nem do bem que me fizeram, nem do mal

Pra mim, tanto faz

Não me arrependo de nada

Porque a minha vida

As minhas alegrias, hoje,

Começam com você."

Non, rien de rien,

Non, je ne regrette rien

Ni le bien qu'on m'a fait, ni le mal

Etc. 


\section{Conclusion}

Cette étude nous a permis d'avancer des arguments nouveaux et concluants à l'appui de la thèse défendue dans Andrade (2015) et Andrade (2016) selon laquelle la chanson dialogique de Maria Bethânia représente un genre du discours autonome. Elle se caractérise par l'hybridité au niveau énonciatif et discursif, se jouant de l'altérité des identités, des langues, des cultures et des discours, avec le concours de la mémoire affective et cognitive, individuelle et collective des destinataires.

Nous avons montré que la méthode suivie pour l'analyse de notre corpus, articulant des approches énonciative et discursive, à travers notamment les notions de circulation de discours, de paratopie, de scénographie et d'effacement énonciatif, non seulement s'est avéré efficace pour les objectifs poursuivis mais qu'il peut inspirer de nouvelles recherches autour de la chanson populaire et d'autres arts.

En ce qui concerne plus particulièrement la chanson dialogique Sou eu mesmo, o trocado- Non je ne regrette rien, nous avons fait ressortir sa fonction (ré)novatrice des sens et des formes vis-à-vis des circulants relayés: le poème de Pessoa Sim, sou eu, eu mesmo, tal qual resultei de tudo et la chanson Non, je ne regrette rien d'Édith Piaf avec paroles de Michel Vaucaire et musique de Charles Dumont.

Nous avons vu que cette rénovation est obtenue, au niveau discursif, par une scénographie construite sur un double processus de dramatisation du poème de Pessoa au début, et de récitation en portugais de la chanson de Piaf sous forme de journal intime dramatisé à la fin, les deux bouts étant reliés par le thème et la musique instrumentale de Non, je ne regrette rien. Au niveau énonciatif, elle est obtenue par une stratégie de brouillage énonciatif rendant l'énonciateure de la chanson dialogique apte à prendre en charge les propos et les points de vue du poème de Pessoa et de la chanson de Piaf, en neutralisant les différences de genre, de langue et de culture, d'espace et de chronologie de ceux-ci. Parmi les effets pragmatiques de ce brouillage énonciatif, nous avons mis en avant celui de la posture surassertive échoénonciative au moyen de laquelle la chanteuse brésilienne vise (et décroche) la reconnaissance de son magistère artistique et moral.

\section{Références}

ANDRADE, Rafael Batista. A (auto)narrativa de vida na construção da canção polifônica Quem é essa agora/Pra rua me levar, de Maria Bethânia. Entremeios (Seção Estudos), Universidade do Vale do Sapucaí, Pouso Alegre (MG), v. 13, p. 87-109, 2016.

ANDRADE, Rafael Batista. Semiótica, éthos e gêneros de discurso nas canções-poemas de Maria Bethânia. Curitiba: CRV, 2015.

ANDRADE, Rafael Batista. Apesar de você (quem?): a produção de sentidos orientada por Maria Bethânia. XI Seminário Internacional em Letras: linguagens e práticas socioculturais, Santa Maria, 2011. p. 1-9.

BAKHTINE, Mikhaïl. Esthétique de la création verbale. Paris: Éditions Gallimard, 1984.

BETHÂNIA, Maria. Abraçar e agradecer. Sarapuí, Biscoito Fino, 2016.

BETHÂNIA, Maria. Imitação da vida. Guarulhos, EMI Music Brasil, 1997.

CAMPOS, Álvaro. Poemas de Álvaro de Campos. São Paulo: Companhia Editora Nacional, 2008.

DUMONT, Charles. Non, je ne regrette toujours rien. Calmann-Lévy, 2012.

LÓPEZ MUÑOZ, Juan Manuel. La circulation des travaux de Peytard en Espagne: lieux, temps, personnes, manières, fins. Jean Peytard, syntagmes et entailles. Limoges: Éditions Lambert-Lucas, 2014. p. 139-155.

LÓPEZ MUÑOZ, Juan Manuel. Les territoires du discours: circulation, exclusion et espaces virtuels (l'e-territorialité). In: KOHLER, Héliane; LOPEZ MUÑOZ, Juan M. (éds.) Exterritorialité, énonciation, discours. Berne: Peter Lang, 2010. p. 17-37.

LÓPEZ MUÑOZ, Juan Manuel. Agents et fonctions de la circulation des discours dans les Lais de Marie de France. L'information grammaticale, n. 118, 2008, p. 27-32. 
LÓPEZ MUÑOZ, Juan Manuel; MARNETTE, Sophie; ROSIER, Laurence. Les enjeux du dire circulaire. Monografías de Çédille, 1 (La circulation des discours: médias, mémoire et croyances), 2010. p. 9-16.

LÓPEZ MUÑOZ, Juan Manuel; MARNETTE, Sophie; ROSIER, Laurence (éds.). Vers une théorie de la circulation discursive. La circulation des discours. Les Éditions Nota bene, 2009.

LÓPEZ MUÑOZ, Juan Manuel; MARNETTE, Sophie; ROSIER, Laurence (éds.). Dans la jungle des discours: genres de discours et discours rapporté. Cadix: Presses de l'Université de Cadix, 2006. https://doi.org/10.3917/tl.052.100

LÓPEZ MUÑOZ, Juan Manuel; MARNETTE, Sophie; ROSIER, Laurence (éds.). Le discours rapporté dans tous ses états. Paris: L'Harmattan, 2004.

LÓPEZ MUÑOZ, Juan Manuel; ROMERAL, Francisca. Discours permanents, discours enco-énonciation et et écho-énonciation dans les entretiens: la pratique de l'autocitation chez Annie Ernaux. Travaux de Linguistique, 52, p. 85-100, 2006.

MAINGUENEAU, Dominique. Trouver sa place dans le champ littéraire. Paratopie et création. Louvain-la-Neuve: Academia, 2016.

MAINGUENEAU, Dominique. Les phrases sans texte. Paris: Colin, 2012.

MAINGUENEAU, Dominique. Quelques implications d'une démarche d'analyse du discours littéraire. COnTEXTES, 2006.

MAINGUENEAU, Dominique. Le Discours littéraire. Paratopie et scène d'énonciation. Paris: Armand Colin, 2004.

MAINGUENEAU, Dominique. Genèse du discours. Bruxelles-Lièges: Mardaga, 1984.

MOISÉS, Carlos Felipe. Roteiro de leitura: poemas de Álvaro de Campos. São Paulo: Ática, 1998.

PAVEAU, Marie-Anne. Interdiscours et intertexte. PUF Presses universitaires de FrancheComté. Annales littéraires de l'université de Franche-Comté. Linguistique et littérature: Cluny, 40 ans après, Oct 2008, Besançon, France, 2010. p. 93-105.

PESSOA, Fernando. Poèmes d'Ávaro de Campos. Traduit du portugais par Patrick Quillier avec la participation de Maria Antónia Câmara Manuel. Paris: Christian Bourgois éditeur, 2001.

RABATEL, Alain. Perspective et point de vue. Communications, v. 85, n. 2, 2009. p. 23-35. https://doi.org/10.3917/commu.085.0023
RABATEL, Alain. L'effacement énonciatif et ses effets pragmatiques de sous et de surénonciation. In: Formes et stratégies du discours rapporté: approche linguistique et littéraires des genres de discours, LOPEZ-MUÑOZ, J. M.; MARNETTE, S.; ROSIER, L. (éds.) Estudios de Lengua y Literatura francesas 14, Université de Cadix, 2003. p. 33-61.

VION, Robert. Effacement énonciatif et stratégies discursives. In: De Mattia, M. et Joly A (éds.), De la syntaxe à la narratologie énonciative. Paris: Ophrys, 2001. p. 331-354.

Recebido em 17/01/2018.

Aceito em 20/04/2018. 